\title{
Influences to ADHD Problem Recognition: Mixed-Method Investigation and Recommendations to Reduce Disparities for Latino Youth
} \author{
94143, Fax: 415-476-7170 \\ Jocelyn Meza, M.A., \\ University of California Berkeley \\ Yuanyuan Jiang, PhD, \\ UCSF \\ Eva Jimenez Araujo, PhD, and \\ University Autonomous of Sinaloa \\ Linda Pfiffner, PhD \\ UCSF
}

Lauren M. Haack, Ph.D. [Clinical Psychology Fellow], University of California, San Francisco (UCSF), 401 Parnassus Avenue G06, San Francisco, CA

\section{Abstract}

ADHD problem recognition serves as the first step of help seeking for ethnic minority families, such as Latinos, who underutilize ADHD services. The current mixed-method study explores underlying factors influencing recognition of ADHD problems in a sample of 159 school-aged youth. Parent-teacher informant discrepancy results suggest that parent ethnicity, problem domain, and child age influence ADHD problem recognition. Emerging themes from semi-structured qualitative interviews/focus groups conducted with eighteen Spanish-speaking Latino parents receiving school-based services for attention and behavior concerns support a range of recognized ADHD problems, beliefs about causes, and reactions to ADHD identification. Findings provide recommendations for reducing disparities in ADHD problem recognition and subsequent help seeking.

\section{Keywords}

ADHD; Latinos; disparities; mixed-method; evaluation

Compliance with Ethical Standards: Conflict of Interest: Dr. Lauren Haack declares that she has no conflict of interest. Jocelyn Meza declares that she has no conflict of interest. Dr. Yuanyuan Jiang declares that she has no conflict of interest. Dr. Eva Jimenez Aurajo declares that she has no conflict of interest. Dr. Linda Pfiffner declares that she has no conflict of interest.

Ethical approval: All procedures performed in studies involving human participants were in accordance with the ethical standards of the institutional and/or national research committee and with the 1964 Helsinki declaration and its later amendments or comparable ethical standards. This article does not contain any studies with animals performed by any of the authors.

Informed consent: Informed consent was obtained from all individual participants included in the study. 
Attention-deficit/hyperactivity disorder (ADHD) is among the most commonly diagnosed psychiatric conditions for children in the United States (U.S.; American Psychiatric Association, 2013; Kessler et al., 2006); however, ethnic minority youth, such as Latinos, show disproportionally low rates of ADHD diagnoses and service utilization (Leslie \& Wolraich, 2007; Pastor \& Reuben, 2005; Stevens, Harman, \& Kelleher, 2005). Importantly, a recent meta-analysis of over 100 studies incorporating ADHD reports from multiple informants failed to uncover prevalence discrepancies based on country or region of the world (Willcutt, 2012). Accordingly, ADHD is a universal disorder, although some of the features appear to manifest dissimilarly to different raters (Faraone, Sergeant, Gillberg, \& Biederman, 2003; Hinshaw \& Scheffler, 2014; Willcutt, 2012; Willcutt et al., 2012). Given the strong neurological and biological substrates of ADHD and emerging evidence of equitable risk for all youth across cultures (Hinshaw \& Scheffler, 2014; Faraone et al., 2003; Rutter \& Nikapota, 2002), investigation of why ethnic/racial disparities in ADHD diagnosis and treatment occur as early as kindergarten entry and persist until later ages is warranted (Morgan et al., 2013).

The ADHD Help-Seeking Behavior Model (Eiraldi, Mazzuca, Clarke, \& Power, 2006) was developed to elucidate predictors of inequalities in ADHD diagnosis and treatment utilization for ethnic minority youth. This model describes four stages in help-seeking, with each stage providing unique opportunities for identifying barriers and culturally sensitive solutions to reduce disparities in treatment utilization. The first stage, Problem Recognition, requires perception of a child's behaviors as problematic in order for families to make the active decision to seek help (second stage), select services (third stage), and utilize services (fourth and final stage; Eiraldi et al., 2006). Latino families, in particular, appear at-risk for various barriers to ADHD problem recognition. To understand and combat this disparity, researchers have identified several factors that contribute to the ADHD problem recognition process, both contextual and cultural in nature (Alvarado \& Modesto-Lowe, 2016; Gerdes, Lawton, Haack, \& Schneider, 2014; Eiraldi et al., 2006; Rothe, 2005; Power, Eiraldi, Clarke, Mazzuca, \& Krain, 2005; Reardon et al., 2017).

To begin, ADHD expression fluctuates across the lifespan (Faraone et al., 2015; Ramtekkar, Reiersen, Todorov, \& Todd, 2010; Todd, Huang, Todorov, Neuman, Reiersen, Henderson, \& Reich, 2008); thus, child age may influence how ADHD problems are recognized (Bussing, Zima, Gary, \& Garvan, 2003; Eraldi, Mazzuca, Clarke, \& Power, 2006). In addition, ratings of ADHD may differ depending on the problem area of interest, such as ADHD symptom cluster or impairment domain. For example, in contrast to inattentive symptoms which generally persist throughout the lifespan, hyperactive-impulsive symptoms often become less apparent or intense as a child matures (American Psychiatric Association, 2013; Biederman, Mick, \& Faraone, 2000; Faraone et al., 2015; Turgay et al., 2012). Additionally, although individuals with ADHD experience persistent impairment in domains of educational achievement, interpersonal relations, and/or emotion regulation, the manifestation of impairment may differ as expectations evolve over a person's lifetime (Biederman et al., 2012; Daley \& Birchwood, 2010; Faraone et al., 2015; Hinshaw, Owens, $\&$ Zalecki, 2012; Turgay et al., 2012). Thus, contextual factors such as child age and problem domain assessed can influence ADHD problem recognition. 
Cultural factors also influence problem recognition, including differential ADHD knowledge, attitudes about psychopathology, and developmental expectations for child behavior across populations (Alvarado \& Modesto-Lowe, 2017; Eraldi et al., 2006; Faraone et al., 2015; Power et al., 2005; Reardon et al., 2017). Specifically, despite the strong support for neurological and genetic etiology of ADHD, many ethnic minority parents do not identify biological causes for child disorders, such as ADHD (Yeh et al., 2004; Lawton, Gerdes, Haack, \& Schneider, 2014). Additionally, ethnic minority parents may be unaware or misinformed about the persistent nature of ADHD, instead believing that the problems will disappear on their own as the child ages (Alvarado \& Modesto-Lowe, 2017). A failure to regard ADHD as a chronic, biological disorder may result in the perception that symptoms are not abnormal and/or do not warrant professional help (Bussing, Gary, Mills, \& Garvan, 2003). This perception may be exacerbated by negative cultural views about psychopathology and mental health services, as well as differential cultural standards for appropriate versus inappropriate child conduct (Alvarado \& Modesto-Lowe, 2017; Eraldi et al., 2006; Power, Eiraldi, Clarke, Mazzuca, \& Krain, 2005). For example, ethnic minority parents may attribute ADHD behaviors to typical child development and feel they are being unfairly targeted when ADHD referrals are given by teachers or providers of a different race (Alvarado \& Modesto-Lowe, 2017).

The Latino population has been deemed particularly vulnerable to ADHD problem recognition barriers for various reasons (Haack, Gerdes, Lawton, \& Schneider, 2014; Rothe, 2005). In addition to general contextual factors (such as child age and problem domain assessed), one must consider socioeconomic influences to problem recognition in Latino youth, as Latino families are disproportionately impoverished and underinsured in the U.S. (DeNavas-Walt, Proctor, \& Smith, 2009; Zambrana \& Carter-Pokras, 2004). For example, Latino youth appear to receive fewer health-care and follow-up visits with physicians than Caucasian youth (Stevens, Harman, \& Kelleher, 2005), thus providing fewer opportunities for health care professionals to inquire about ADHD problems. Furthermore, given the lower rates of parent-teacher communication and family-school involvement in Latino compared to non-Latino communities (Wong \& Hughes, 2006), discussion of child behavior between teachers and parents may be especially limited for Latino youth, especially if teachers and parents speak different languages.

Knowledge about ADHD and its causes also appears limited in Latino population, as Latinos are less likely than Caucasians to report familiarity with ADHD in large national surveys (McLeod, Fettes, Jensen, Pescosolido, \& Martin, 2007; Rothe, 2005). In qualitative research, many Latino families describe beliefs that ADHD behaviors are caused by family factors, such as a lack of parent attention or discipline, and are likely to go away on their own (Gerdes et al., 2014). Additionally, Latino parents may have difficulty accepting their child's behavior problems (Gerdes et al., 2014), especially when behaviors are perceived as a lack of manners or disrespect for authority (Perry et al., 2005). In contrast, Latino parents may be less concerned about ADHD symptomology, particularly in the hyperactivity-impulsivity domain, and instead may believe these behaviors are a normal aspect of child development (Arcia \& Fernánde, 2003; Gerdes, Lawton, Haack, \& Hurtado, 2013). 
Orientation to cultural values may help explain Latino parents' beliefs about the cause and burden of various ADHD behaviors. Collectivistic values, such as personalismo (i.e., emphasis on warm, interpersonal closeness), familismo (i.e., emphasis on family connectedness and loyalty), respeto (i.e., emphasis on obedience and compliance to authority), spiritualismo (i.e., religiosity), and traditional gender roles or machismo (i.e., emphasis on masculine pride and feminine submissiveness) are particularly relevant to perceptions about and treatment of child behavior problems (Alvarado et al., 2016; Barker, Cook, \& Borrego Jr., 2010; Lawton et al., 2014; Rothe, 2005). For example, valuing family loyalty, traditional gender roles, and spiritualism may be discordant with perceptions that hyperactive/impulsive symptoms are abnormal and indicative of a concerning, biological disorder (e.g., "Sure, my son is spirited, but that is the way God made him, and I actually love those qualities in him! After all, boys will be boys" ${ }^{\text {"1 }}$. In contrast, these values along with respect for authority may align with perceptions that impairment related to noncompliance with rules and instructions (and in particular from male family members) is problematic (e.g., "Children must respect adults, especially their fathers. If they don't, it reflects poorly on the family." ${ }^{\prime \prime}$ ). Indeed, previous research has uncovered associations between parent endorsement of cultural values with ratings of ADHD symptom severity (Schmitz \& Velez, 2003), tolerance for their children's behavior problems (Halgunseth, Ispa, \& Rudy, 2006; Roberts, Alegria, Roberts, \& Chen, 2005), and etiological beliefs about the cause of ADHD (Lawton et al., 2014).

In summary, Latino families appear at-risk for various barriers to ADHD problem recognition, which serves as the first step of help-seeking and thus likely contributes to disproportionately low rates of ADHD identification and service utilization for Latino youth. Given that health disparities are embedded in a complex interplay of contextual and cultural issues, quantitative research investigating discrepancies supplemented by qualitative research providing in-depth understanding of vulnerable populations' needs is called for (Curry, Nembhard, \& Bradley, 2009; Stewart, Makwarimba, Barnfather, Letourneau, \& Neufeld, 2008; Sullivan-Bolyai, Bova, \& Harper, 2005). Further, mixed-method exploration of underlying factors influencing ADHD problem recognition could inform culturally competent mental health policy, practice, and program development for Latino families.

Methods for investigating problem recognition informant discrepancies have evolved over the years. Although it may seem logical to examine difference scores (for example, the difference between parent and teacher ratings as a means of identifying over- or underreporting), there are various limitations to difference scores (Laird \& LaFleur, 2014; Laird \& LaFleur, 2016; Laird \& Reyes, 2012; Laird \& Weems, 2011). To begin, an association between the difference score and the outcome/informant characteristics may actually represent an association between the outcome and one but not both informant reports, such that unequal variances between component scores may lead to misleading associations. Another weakness of difference scores is that using such a score is equivalent to constraining the coefficients on teacher and parent scores to be equal in magnitude but opposite in sign, which is incongruent with theory. Thus, polynomial regressions are currently recommended

\footnotetext{
${ }^{1}$ Note. Quotes were derived to provide examples of concepts; they do not represent actual parent quotations.
} 
to evaluate problem recognition informant discrepancies (Laird \& LaFleur, 2014; Laird \& LaFleur, 2016; Laird \& Reyes, 2012; Laird \& Weems, 2011). Polynomial regression analyses allow for consideration of the unique perspectives of two informants (e.g., parents versus teachers) and thus provide information about the direction and degree of informant disagreement. This information can be used to examine if greater parent-teacher discrepancies predict the probability of being of Latinos status, as well as if discrepancies between raters (parent or teacher) differ depending on the outcome being rated.

\section{Current Study}

Our goal of the current study was to investigate influential factors to ADHD problem recognition in a sample of Latino and non-Latino youth receiving school-based services for attention and behavior concerns (i.e., the Collaborative Life Skills [CLS] program; source masked for blind review) in order to understand disparities and provide recommendations for culturally-sensitive care. First, we examined differences in parent ${ }^{2}$ and teacher reports of child ADHD symptoms and impairment (Part One). Based on previous research suggesting that Latino parents under-recognize ADHD symptoms compared to impairment (Gerdes et al., 2013), we hypothesized that ADHD problem recognition would be influenced by parent ethnicity, such that inconsistency in parent-teacher reports of ADHD symptoms would predict Latino family status. In contrast, we predicted Latino families would not exhibit parent-teacher discrepancies in their report of child impairment. We also conducted exploratory analyses to examine parent-teacher discrepancies in ratings based on child age and symptom cluster assessed (i.e., inattentive versus hyperactive-impulsive). To consider whether findings indeed are driven by informant reporting rather than differences in crosssituational child behavior, we also examined discrepancies between parent and teacher ratings with observed child behavior in the classroom for Latino and non-Latino youth.

Next, to help explain quantitative results, we uncovered emerging themes in qualitative feedback from a subsample of Latino parents participating in the four Spanish-language cohorts of CLS (Part Two). We hypothesized that emerging themes would support previous research suggesting that Spanish-speaking Latino parents: a) identify impairment as concerning, b) recognize a range ADHD etiological beliefs, including family factors in addition to biological/physical causes, and c) describe reactions to ADHD problem identification reflective of contextual and cultural factors relevant to Latinos in the U.S.

\section{Method}

\section{Participants}

For Part One of the current study, participants included 159 children in grades second through fifth across 27 schools in an urban public school district. Boys constituted close to three-quarters of the sample (72\%); children averaged 8.36 years of age (standard deviation $=1.10)$. Regarding race/ethnicity, 56 (35\%) participants were Hispanic/Latino, 36 (23\%) participants were Caucasian, 28 (18\%) participants were Asian-American, 12 (8\%)

\footnotetext{
${ }^{2}$ We use the term "parent" throughout this manuscript; however, we acknowledge that several extended family members (e.g., grandmothers) participated as primary caregivers in lieu of parents.
} 
participants were African-American, and 27 (17\%) participants were of mixed ethnicity. Thirty (19\%) children spoke Spanish as their primary language. The breakdown of $35 \%$ Latino versus $65 \%$ non-Latino youth generally is representative of the school district and greater area (location masked for blind review). Among our Latino participants whom disclosed country of origin, 66\% reported Mexico and 33\% reported another Latin American country (i.e., Guatemala, Nicaragua, Honduras, or El Salvador). Each child had one parent designated as the "primary caregiver" complete all questionnaires and measures. More complete demographic information for Latino and non-Latino parents and children may be found in Table 1.

To become enrolled in the study, children needed to have at least six inattention symptoms and/or at least six hyperactive/impulsive symptoms endorsed on the Child Symptom Inventory (CSI; Gradow \& Sprafkin, 2002) by the parent -or- teacher as occurring often or very often; cross-situational impairment (home and school), documented as a score of at least three in at least one domain of functioning on parent and teacher Impairment Rating Scales (IRS; Fabiano et al., 2006); Full-Scale Intelligence Quotient equivalent to higher than 79 on the Wechsler Abbreviated Scale of Intelligence (WASI; Wechsler, 2011) to ensure children would be able to understand and effectively participate in group sessions; a caretaker available to participate in treatment; and a primary classroom teacher who agreed to participate in the classroom component. More detailed information about the CLS Randomized Controlled Trial (RCT) can be found in the primary outcomes paper (source masked for blind review).

We examined group differences between Latinos and non-Latinos in Part One of the current study on demographic factors and reports of ADHD symptoms and impairment via independent samples $t$-tests. Significant differences emerged for several characteristics between Latinos and non-Latinos in our study (Table 1). There were significant, but modest, differences on the WASI FSIQ between Latinos and non-Latinos in our study. Specifically, Latino children demonstrated lower cognitive abilities on the WASI FSIQ; however, both Latino and non-Latino WASI FSIQ mean scores fell solidly within the Average range. Latino children were more likely to live in single-parent households and receive public assistance, such as social security income. Household incomes and primary parent education attainment was significantly lower for Latino compared to non-Latino families in our sample. Importantly, no group differences in parent or teacher reports of ADHD symptoms or impairment emerged between Latino and non-Latino groups (Table 2). Comparing ADHD symptom clusters within rater, however, parent-rated inattention severity was higher than parent-rated hyperactivity/impulsivity severity in both groups.

For Part Two of the current study, participants included a subsample of eighteen Latino parents from the four schools designated to receive the CLS program in Spanish (out of 27 total schools participating in the CLS trial). Out of the 24 total parents in the four designated Spanish-speaking CLS cohorts, eighteen parents participated in a focus group $(\mathrm{n}=11$ across three focus groups) or individual interview $(\mathrm{n}=7)$. Briefly, participating parents were predominantly mothers with varying levels of education and employment status; children were mostly boys ranging in age from seven to ten. No demographic differences emerged when comparing Spanish-speaking parents who did and did not complete focus groups or 
interviews. Given the importance of considering potential cultural differences between Spanish-speaking parents who did versus did not provide qualitative data, we also investigated for group differences on measures of acculturation between groups (i.e., the Acculturation Rating Scale for Mexican Americans [ARSMA-II; Cuellar, Arnold, \& Maldonado, 1995] and the Mexican American Values Scale for Adolescents and Adults [MACVS; Knight et al., 2010]). Our sample of Latino parents participating in the Spanishspeaking cohorts of CLS reported higher ratings of affiliation to Latino value and behavior orientation compared to Anglo value and behavior orientation with no significant group differences between those who did and did not provide qualitative data (Table 3).

\section{Procedure}

Children across schools in the blinded for review Unified School District were identified as potential participants for the study via school staff based on difficulties with attention, hyperactivity/impulsivity, and impairment. Next, school mental health providers contacted families and teachers of identified students to discuss the program and interested parents provided Releases of Information to allow communication with the clinical research team. Lastly, clinical research team members completed telephone screenings with parents and teachers regarding the child's academic, social, and behavioral functioning. For more details regarding recruitment procedures, see (source masked for blind review).

Parents and teachers completed a series of questionnaires for Part One of the study, including measures of child behavior, as well as parent and family functioning, as part of the initial screening and assessment procedure. Participants provided informed consent and children provided assent. The Committee on Human Research at the participating university approved all study procedures.

For Part Two of the current study, Spanish-speaking Latino parents responded to an ad hoc semi-structured focus group or interview; parents provided consent to participate and be video recorded. Parents participated in the focus group/interview after receiving the CLS intervention and providing follow-up data (i.e., ten from CLS and eight from BAU waitlist control condition). Specifically, the ten CLS parents responded to the focus group/interview immediately after receiving CLS and providing follow-up data; the eight BAU parents responded to the focus group/interview after being held on the waitlist, providing follow-up data, and then receiving the CLS intervention. Our decision to solicit qualitative feedback from the Spanish-speaking CLS cohorts was intentional in order to obtain in-depth information from a traditionally over-looked group in clinical research (i.e., non-English speaking parents). Keeping in mind cultural differences and sensitivity to parents' potential preferences for keeping family matters private, we offered participants the option of engaging in individual interviews if they did not want to provide feedback in a focus group. Participants were video recorded to allow for time-coded transcription and coding in Transana Multiuser Version 3.2 software (Woods, 2016), as well as consideration of nonverbal cues and lip-reading to maximize accurate transcription. Study objectives were described to participants prior to the focus groups and interviews; specifically, it was explained that researchers hoped to obtain information about help-seeking for attention/ 
behavior concerns and recommendations for implementing services with Spanish-speaking Latino families.

Two bilingual moderators conducted each focus group and interview: one moderator was a native Spanish speaker and one moderator was a native English speaker. One moderator led the discussion, asking each question from the Experiences with CLS Qualitative Outline in order with both moderators providing prompts and clarification as needed. We created the Experiences with CLS Qualitative Outline focused on the four stages of the ADHD Help Seeking Behavior Model: Problem Recognition, Decision to Seek Help, Service Selection, and Service Utilization (Eiraldi et al., 2006). Within the Problem Recognition phase of interest to the current study, we focused questions on identification of behaviors, causes of behaviors, and reactions to identification of behaviors (see Table 4 for questions and prompts relevant to problem recognition). We conducted three focus groups, each containing three to four parents who had participated together in the CLS parent group; focus groups lasted between nineteen and 48 minutes (average $=32$ minutes) with length depending on the amount of detail provided by the respondents. All focus groups were conducted in Spanish. We conducted seven individual interviews ranging from twelve to 40 minutes (average $=24$ minutes) and all but one was conducted in Spanish.

\section{Measures}

Child Symptom Inventory (CSI; Gradow \& Sprafkin, 2002)—The CSI is a behavior rating scale used to screen emotional and behavioral disorders as outlined by DSM-IV criteria in five to twelve year old children. The parent checklist contains 97 items and the teacher checklist contains 77 items. Symptoms are rated on a 4-point scale $(0=$ never to $3=$ very often) and are considered to be present when they are rated as occurring often or very often (i.e., 2 or 3 on the 4-point, 0-3 scale). We used parent and teacher composite severity scores on the Inattention and Hyperactivity-Impulsivity domains as outcome variables of interest. The Inattention and Hyperactivity-Impulsivity scales have normative data, acceptable test-retest reliability, and high predictive and concurrent validity for categorical diagnosis of ADHD (Gadow \& Sprafkin, 1994).

Impairment Rating Scale (IRS; Fabiano et al., 2006)—We used the IRS to assess impairment across settings and areas of functioning (i.e., academics and peer relations). We used both parent and teacher versions. The IRS uses a seven-point Likert scale to evaluate the degree to which the child has problems that warrant treatment, ranging from 0 (i.e., "no problem; does not need treatment or special services") to 7 (i.e., "extreme impairment; definitely needs treatment or special services.") For children ages four through twelve, the IRS has shown good psychometric properties and has empirically derived cutoff points (Fabiano et al., 2006). One item inquires about the overall severity of impairment (i.e., Please mark an " $X$ " on the following line at the point that you believe reflects the overall severity of this child's problem in functioning and overall need for treatment with item response anchors of No Problem/Definitely does not need treatment for special services to Extreme Problem/Definitely needs treatment or special services). We used this overall severity item score for our analyses. 
Behavioral Observation of Students in Schools (BOSS; Shapiro, 2004)-We used the BOSS to assess direct classroom observations. The BOSS measures both task engagement (i.e., active engagement and passive engagement) and off-task behavior (i.e., off-task motor behaviors, off-task verbal behaviors, off-task passive behaviors). Task engagement is rated using momentary time sampling at the beginning of each 15 -second interval, while off-task behaviors are coded during the remainder of each interval (partial interval method). In terms of training, each coder first learned about the BOSS system through our BOSS instructional training session. This training session provided background for the CLS study, the purpose of observing students, the goal of reliability, the logistics of scheduling and attending observations, the definitions of BOSS codes, and how these codes should be assessed. Coders then partnered up with an already-established coder to practice coding on two to three separate occasions during classroom academic periods. Each individual needed $80 \%$ agreement or a minimum kappa of .7 for both Task Engagement and Off-Task Behavior codes with the well-established coder before he/she was able to code independently. Two coders concurrently coded sessions for approximately $30 \%$ of our observations, demonstrating inter-rater reliability of at least 0.7 kappa (80\% agreement; kappa of .83 for TE and .72 for OTB). For this study, we used composite scores to capture task engagement and off-task behavior averaged across observations. Previous studies using these BOSS codes report high inter-observer agreement and ability to distinguish between typically-developing children and children with ADHD (DuPaul et al., 2004). In our dataset, the BOSS subscales of task engagement and off-task behavior were significantly correlated with teacher-rated ADHD symptoms, $r(155)=-.17, p=.03$ for task engagement, and $r(155)$ $=.16, p=.04$ for off-task behavior.

Participant Characteristics-Each parent in the study completed a family history form to gather sociodemographic information, including; child age, child and parent race/ ethnicity, parent's level of education, and members living in the household. Latino parents participating in the 4 Spanish-speaking CLS cohorts also responded to questionnaires inquiring about affiliation to cultural value and behavior orientation (i.e., MACVS and ASRMA-II; see descriptions below). We gathered this information to allow for consideration of acculturation - a particularly salient characteristic for U.S. Latinos-- when investigating our Spanish-speaking Latino parents' problem recognition and experiences with CLS services.

\section{Mexican-American Cultural Values Scale for Adolescents and Adults} (MACVS; Knight et al. 2010)—The MACVS is a 50-item measure of several cultural constructs relevant to traditional Latino and mainstream American cultural value orientation. Items are measured on a 5-point Likert scale ranging from $1=$ not at all to 5=completely. The MACVS has shown good psychometric properties, including adequate reliability construct validity (Knight et al., 2010).

\section{Acculturation Rating Scale for Mexican-Americans-II (ARSMA-II; Cuellar et al.} 1995)—The ARSMA-II is a 30-item self-report measure. It assesses the parallel processes of acculturation to both culture of origin and host culture in terms of behavior orientation, including language use and ethnic interaction. Items are rated as not at all (0) to extremely 
often or almost always (5). The original ARSMA-II frames questions specifically to Mexican-Americans; thus, in order to accommodate all Latino subgroups, the word "Mexican" was changed to "Latino." This method has been used previously and maintains good reliability (e.g., Cronbach's alpha $=.78$; Steidel and Contreras 2003). Strong internal consistencies for the AOS (.83) and LOS (.88), as well as construct validity in a sample of 379 individuals representing five generations, have been reported (Cuellar et al. 1995).

\section{Data Analysis}

We used polynomial regressions to examine problem recognition disparities, as the clinical research field considers polynomial regressions to be the current standard for informant discrepancy research given several limitations of using difference scores (e.g., Laird \& Reyes, 2012; Laird \& LaFleur, 2016). As described in the introduction, according to Laird and Weems (2011), a number of drawbacks render difference scores sub-optimal for testing informant discrepancies, such as unequal variances and constraints on difference score components (Laird \& Weems, 2011). In contrast, regressions with interaction terms are able to retain the different perspectives of two informants and allow for assessing the direction and degree of informant disagreement. These regressions also include quadratic predictors so as not to underestimate the complexity of model fit. Thus, in this study, we used logistic polynomial regressions to examine parent and teacher discrepancies in ratings of ADHD symptoms and impairment in predicting the probability of being of Latino status, as well as discrepancies between raters (parent or teacher) and direct classroom observations in predicting Latino status.

For Part One, we conducted logistic polynomial regressions using IBM SPSS Version 23 (IBM SPSS, 2015) to examine the pattern of parent and teacher ratings of ADHD and impairment in Latino and non-Latino families. In particular, we predicted the probability of Latino status by parent and teacher ratings as individual predictors and their interaction. For significant interactions, we conducted probing of simple slopes. We also examined these polynomial analyses based on parent and teacher ratings of ADHD symptom cluster and child age, as well as for models including direct classroom observations.

For Part Two, we used Transana Multiuser Version 3.2 (Woods, 2016) software to transcribe, code, and analyze the qualitative data. First, we created transcriptions synced with video footage of the interviews/focus groups. Next, we developed a hierarchical coding system based on recurrent concepts from interviews/focus groups and theoretical literature utilizing Thematic Analysis Principles (Creswell \& Clarke, 2007). Codes were considered endorsed based upon both verbal responses and verbal agreement (i.e., if a parent in a focus group said "me too,"this agreement was coded as an endorsed theme corresponding to the original verbal response). Two bilingual clinical researchers (one native English-speaker and one native Spanish-speaker) coded each transcription independently using the hierarchical system; the clinical researchers converged to discuss discrepancies and consulted a third party if needed to resolve discrepancies. The team of coders collaboratively uncovered recurrent themes and the coding system was updated iteratively. Once codes were finalized, we generated individual collection reports to examine the frequency and length of time in which each code was discussed by each parent regardless of response modality (i.e., 
interview or focus group). Given that all qualitative research is influenced by personal and cultural lenses, we made a concerted attempt to discuss when potential biases may be compromising neutrality. Specifically, we documented our decision trail in an effort to uphold transparency of our qualitative coding. In addition, we employed an "expert critique" approach (Sinkovics \& Alfoldi, 2012) which involves the researcher (i.e., first author) asking two others on the team (i.e., co-authors) to examine the data independently and confirm the decision-making process and conclusions made. For this study, we solicited feedback from "auditors" who were familiar with the content area, in this case from multiculturalmultilingual team members.

\section{Results: Part One}

\section{ADHD Symptom Severity}

We conducted logistic polynomial regressions with parent-rated ADHD symptoms, teacherrated ADHD symptoms, and their interaction and quadratic terms as predictors of the probability of Latino status. There was a significant quadratic main effect of parent-rated ADHD severity $(\beta=.53, S E=.20, p=.01)$ qualified by a significant parent- by teacherrating interaction in predicting Latino status $(\beta=-.45, S E=.23, p=.046$; see Figure 1). Probing of simple slopes demonstrated that a discrepancy in the direction of high teacherreported ADHD and low parent-reported ADHD was related to a higher likelihood of being Latino $(\beta=-.55, S E=.27, \mathrm{p}=.04)$. The slope representing a discrepancy in the opposite direction (high parent-reported ADHD and low teacher-reported ADHD in predicting higher Latino status) was non-significant ( $\beta=-.46, S E=.32, p=.15$ ); visual inspection suggested a nonsignificant trend toward families being more likely to be Latino when there is an inconsistency between parent and teacher reports of ADHD.

ADHD symptoms based on symptom cluster and age-Similar regressions were carried out breaking down the results by ADHD symptom cluster and age. We based age analyses on a mean split within those either younger or older than 8.36 years (younger $n=$ 86 , older $n=73)^{3}$. When the inattention symptom cluster was rated for younger children, there was a significant quadratic main effect of parent-report $(\beta=-.57, S E=.26, p=.03$ ) with visual inspection suggesting a curved relationship in which both high and low levels of parent-reported inattention are associated with a higher probability of Latino status. When inattention symptoms were rated for older children, there was a significant linear main effect of teacher-ratings ( $\beta=.68, S E=.31, p=.03$ ) suggesting that higher teacher-rated inattention symptoms were associated with higher probability of Latino status. There were no significant parent- by teacher-rating interactions for the inattention symptom cluster across ages.

For hyperactivity/impulsivity symptoms and younger children, there was a non-significant trend for a quadratic main effect of parent-ratings $(\beta=.60, S E=.34, p=.08)$, which was qualified by a significant parent- by teacher-rating interaction in predicting Latino status ( $\beta$ $=-.87, S E=.41, p=.03$ ). Simple slopes (see Figure 2) indicated that Latino families were

\footnotetext{
${ }^{3}$ Data were missing for up to 8 participants depending on analysis; only approximately $2 \%$ of data was missing for primary variables.
} 
more likely to have high parent-ratings and low teacher-ratings $(\beta=-1.27, S E=.58, p=.03)$ as well as low parent-ratings and high teacher-ratings $(\beta=-1.34, S E=.51, p=.009)$. No other effects were found for the hyperactivity/impulsivity symptom cluster across ages.

\section{Impairment}

We conducted similar logistic polynomial regressions with parent and teacher ratings of impairment. Results (see Figure 3) showed a linear main effect such that higher parentreported impairment was related to a higher probability of Latino status, regardless of teacher-ratings $(\beta=.79, S E=.22, p<.001)$, and a quadratic main effect $(\beta=.59, S E=.14$, $p<.001$ ) showing a curved relationship in which higher parent-reported impairment is also related to a higher probability of Latino status. There was no significant parent- by teacherrating interaction in predicting Latino status.

Impairment based on age-We conducted similar polynomial regressions broken down by age (i.e., those younger and older than 8.36 years old). For younger children, there was a non-significant trending linear main effect of parent-reported impairment $(\beta=.56, S E=.31$, $p=.08)$ and a significant quadratic main effect of parent-rated impairment $(\beta=.60, S E=$. $21, p=.005)$ qualified by a significant parent- by teacher-rating interaction in predicting Latino status (see Figure $4 ; \beta=-.77, S E=.38, p=.04$ ), indicating that Latino families were more likely to have high parent-report and low teacher-report of impairment ( $\beta=1.33, S E$ $=.50, p=.008)$. For older children, there was a significant linear main effect of parentreported impairment ( $\beta=1.13, S E=.36, p=.002)$, as well as a significant quadratic main effect of parent-reported impairment $(\beta=.68, S E=.31, p=.03$ ), both suggesting that higher parent-reported impairment is related to a higher probability of Latino status. There were no significant parent- by teacher-rating interactions. We found a non-significant trend for a linear main effect in teacher-ratings $(\beta=.63, S E=.37, p=.09)$, such that Latino families were more likely to have higher teacher-ratings as well.

\section{ADHD Symptom Severity and Direct Classroom Observations}

We carried out similar polynomial analyses pairing both of the direct classroom observation subscales of the BOSS (Task Engagement and Off-Task Behavior) as predictors alongside either parent-or teacher-ratings of ADHD severity. No significant main effects or interactions were found. More specifically, there were no main effects of Off-Task Behavior $(\beta=.32, S E=.55, p=.56)$ or effects of its interaction $(\beta=.25, S E=.54, p=.65)$ with parent-ratings of ADHD severity. There were also no main effects of Task Engagement ( $\beta=$ $-.49, S E=.55, p=.62)$ or effects of its interaction $(\beta=-.31, S E=.57, p=.73)$ with parentratings of ADHD severity. Likewise, there were no main effects of Off-Task Behavior ( $\beta=-$. $25, S E=.60, p=.78)$ or effects of its interaction $(\beta=-.64, S E=.81, p=.43)$ with teacherratings of ADHD severity. There were also no main effects of Task Engagement $(\beta=-.11$, $S E=.59, p=.90)$ or effects of its interaction $(\beta=.29, S E=.72, p=.69)$ with teacherratings of ADHD severity. 


\section{Results: Part Two}

Major themes, sub-themes, and codes endorsed by our subsample of eighteen Spanishspeaking Latino parents for ADHD Problem Recognition, ADHD Etiology, and Reactions to ADHD Identification are presented in Tables 5 through 7. In each table, bolded frequencies indicate the number of parents endorsing at least one code within the theme. Parents could endorse multiple codes per theme and thus, the italicized descriptive statistics indicate the average number of codes identified per theme.

\section{ADHD Problem Recognition}

Three major themes emerged regarding ADHD Problem Recognition: Impairment, Symptoms, and "ADHD/ADD". The Impairment major theme contains three sub-themes: Academic, Social/Emotional, and Home Impairment. The Symptom major theme contains two sub-themes: Inattentive and Hyperactive/Impulsive. Only one parent (6\%) named a diagnosis of "ADHD/ADD". See Table 5 for frequencies of themes, subthemes, and codes, as well as example statements for each code.

Almost all parents (94\%) in our subsample identified at least one code reflecting Impairment. The most common Impairment sub-theme was Social/Emotional Impairment, endorsed by 13 parents (72\%). Within Social/Emotional Impairment, the most common endorsed codes were "doesn't express/show feelings appropriately" (7 parents, 39\%), "distractive, disruptive, and/or bothersome to peers"(4 parents, 22\%), and "ignored, rejected, or teased by peers" (3 parents, 17\%). The next most common Impairment subtheme was Academic Impairment, endorsed by 11 parents (61\%). The most common Academic Impairment codes were "behind academically/low grades"(6 parents, 33\%), "doesn't listen/pay attention in class" (4 parents, 22\%) and "doesn't finish/complete homework" (4 parents, 22\%). Home Impairment was endorsed by slightly less than half of the subsample ( 8 parents, $44 \%$ ). The most common codes reflecting Home Impairment include "doesn't finish/complete homework" (4 parents, 22\%; also reflects Academic Impairment) and "creates stress for caregivers"(3 parents, 17\%).

The majority of parents in our subsample (78\%) identified at least one code reflecting symptoms. Inattention was endorsed by 13 parents (72\%) and Hyperactivity/Impulsivity was endorsed by 5 parents (28\%), consistent with analyses above showing that within the Latino group, inattention was significantly reported more than hyperactivity/impulsivity. The most common codes reflecting Inattention were "easily distracted"( 8 parents, 44\%), "trouble focusing"(6 parents, 33\%), "generally inattentive"(5 parents, 28\%) and "trouble sustaining attention" (5 parents, 28\%). The most common codes reflecting Hyperactivity/Impulsivity were "generally hyperactive/restless"(5 parents, 28\%), "talks excessively"(2 parents, $11 \%$ ) and "behavior problem" (2 parents, $11 \%)$.

\section{ADHD Etiology}

Two major themes emerged regarding ADHD etiology: Biopsychosocial and Sociological/ Spiritual. The Biospychosocial major theme contains six codes: Psychological/Emotional, Familial/Family Discord, Educational, Physical/Biological, Trauma, and Relational Issues. 
The Sociological/Spiritual major theme contains three codes: Cultural Issues/Acculturation, Friends/Peer Group, and Environment/Spiritual/Nature Disharmony. See Table 6 for frequencies of themes and codes, as well as example statements for each code.

The majority of parents in our subsample (16 parents, $89 \%$ ) endorsed codes reflecting Biopsychosocial etiology. The most common codes under this theme were "psychological/ emotional"(14 parents, 78\%), "familial/family discord"(6, 33\%), "educational"(5 parents, $28 \%$ ), and "physical/biological" (5 parents, 28\%). Less than one fourth of our subsample endorsed codes reflecting Sociological/Spiritual etiology. The most common code reflecting this theme was "cultural issues/acculturation," which was endorsed by 3 parents (17\%).

\section{Reactions to ADHD Identification}

Three major themes emerged regarding Reactions to ADHD Identification: Positive Thoughts/Emotions, Negative Thoughts/Emotions, and Thoughts/Emotions Reflecting Culture. See Table 7 for frequencies of themes and codes, as well as example statements for each code. Statements reflecting agreement, contentment, optimism, or hope in the context of the focus group/interview as a whole were coded as Positive Thoughts/Emotions. Statements reflecting struggle, despair, or refusal in the context of the focus group/interview as a whole were coded as Negative Thoughts/Emotions. Statements of thoughts/emotions related to ADHD identification which were not reflective of a positive or negative sentiment in the context of the focus group/interview as a whole, but rather related to culture, were coded as Thoughts/Emotions Reflecting Culture.

Several parents in our subsample $(3 ; 17 \%)$ identified at least one code reflecting Positive Thoughts/Emotions related to ADHD Identification, with the most common endorsed code reflecting "agree there's a problem" (11\%). Just under half of our subsample (6 parents; $44 \%$ ) identified at least one code reflecting Negative Thoughts/Emotions related to ADHD Identification. The most common codes reflecting Negative Thoughts/Emotions were "it was hard/difficult"(4 parents, 22\%), "shock"(2 parents, 11\%), "guilt"(2 parents, 11\%), and “denial”"(2 parents, 11\%). Thoughts/Emotions Reflecting Culture were endorsed by 6 parents (44\%). The codes in this category include "respeto is an important part of $A D H D$ " 4 parents, 22\%), "high activity is normal, especially for boys"(3 parents, 17\%), and "ADHD is unfamiliar in Latino population"(2 parents, 11\%).

\section{Discussion}

Overall, results of the current study indicate that various factors (including parent ethnicity, problem domain, and child age) influence problem recognition, an important stage in ADHD help-seeking for ethnic minority families (Eraldi et al., 2006). Findings extend previous research by examining how parent and teacher inconsistencies in their reports of ADHD symptoms may differ depending on Latino ethnicity status. Furthermore, this study examines qualitative feedback from a subsample of Spanish-speaking Latino parents receiving school-based services in order to better understand how this vulnerable group may experience the problem recognition stage of help seeking. 
To begin, results of the quantitative analyses support the prediction that parent ethnicity influences ADHD problem recognition. In addition, symptom cluster and age range of the child assessed appear to influence ADHD problem recognition. Our findings suggest that Latino families may be more likely than non-Latino families to have parents who underestimate ADHD symptoms compared to teachers. In particular, Latino parents appear to differ from teachers regarding ratings of hyperactivity/impulsivity symptoms for younger children. Latino families in our sample also were more likely to have parents who report higher levels of impairment irrespective of teacher ratings than non-Latino families. More specifically, in younger children, Latino parents in our sample tended to report higher child impairment than teachers, whereas in older children, Latino parents and teachers both tended to have high ratings of impairment.

It is possible that our findings regarding the discrepancy between Latino parent-teacher ratings of younger children are due to the amount of communication between parents and teachers about child behavior. Of note, parent-teacher communication tends to be lower for Latino compared to non-Latino families (e.g., Wong \& Hughes, 2006) and this may be especially true for Spanish-speaking parents of young children. Specifically, the quantity and/or quality of Latino parent-teacher communication may improve as children progress through school and families become more comfortable interacting with school personnel. Subsequently, this comfort with school personnel may enhance parent-teacher agreement on reports of ADHD symptoms and impairment as the child ages. Importantly, the lack of discrepancies between parent or teacher ratings versus observed classroom behavior suggests that discrepancies between parent- and teacher-report indeed are driven by raterbias. These findings support the influence of well-documented ADHD informant discrepancies (Wolraich et al., 2004) to problem recognition and emphasize the need to consider rater bias when evaluating child functioning. Consequently, single-informant evaluation of ADHD should be avoided, especially when working with young children of Latino families.

Emerging qualitative themes from a subsample of Spanish-speaking Latino parents receiving school-based services for youth attention and behavior concerns support a range of recognized ADHD problems, beliefs about causes, and reactions to ADHD identification in the Latino population. Consistent with our prediction, when asked to describe the reason their child was referred for services, almost all Spanish-speaking Latino parents in our subsample described problems related to impairment. Interestingly, the majority of Spanishspeaking Latino parents in our subsample also described problems related to inattention, but only a small minority described problems related to hyperactivity-impulsivity, consistent with within-group descriptive analyses. These findings, albeit preliminary, support previous research suggesting that Latino parents may be most concerned about impairment violating traditional collectivistic values, such as getting along with others or respecting teachers and parents (Arcia \& Fernández, 2003; Gerdes et al., 2013; Gerdes et al., 2014; Perry, Hatton, \& Kendall, 2005). When considering the current study results in context of previous literature, it may be that impairment is more likely impacted by important Latino cultural values and expectations, such as respecto and familismo, than symptomatology (and particularly hyperactivity/impulsivity). Furthermore, impairment may be a more motivating factor for parents to target in treatment, and thus could be leveraged to improve treatment 
enngagement for Latino families. For example, perhaps outreach/recruitment materials highlighting potential improvement in child respect and family harmony would appeal to Latino parents moreso than materials highligthing potential reduction in symptomotology. Additionally, only one Spanish-speaking Latino parent in our subsample described the diagnostic label of ADHD, supporting previous research suggesting Latinos are unfamiliar with diagnostic terminology (Gerdes et al., 2014).

When asked about the cause of these problems, as predicted, the Spanish-speaking Latino parents in our subsample described a range of etiological beliefs. However, in contrast to previous mixed-method research with Latino parents in which family causes are the most commonly identified ADHD etiological belief (Gerdes et al., 2014), most Spanish-speaking Latino parents in our study identified ADHD causes related to psychological/emotional child characteristics. Causes related to the family, education, and/or biology/genetics were described by some Spanish-speaking Latino parents in the current study and a small minority of Spanish-speaking Latino parents described other causes related to culture or acculturation. Previous research has suggested that families may not seek professional help if they do not identify biological/genetic causes for ADHD (Bussing, Gary, Mills, \& Garvan, 2003). Indeed, one can imagine how beliefs about biological/genetic causes would motivate medical help-seeking, whereas beliefs about family causes (such as a lack of parental discipline or attention, as uncovered by Gerdes et al., 2014) may discourage a family from seeking medical help. However, we could posit that families identifying psychological/ emotional causes for ADHD may be willing to seek help from a mental health professional and families identifying educational causes for ADHD may be willing to seek help from the school. Thus, our study in context with previous literature could suggest that psychological and school-based ADHD services may be well-aligned with beliefs about causes in the Spanish-speaking Latino population, and perhaps better aligned than medical or hospitalbased services.

When asked about reactions to ADHD problem identification in their children, emerging themes from our subsample of Spanish-speaking Latino parents reflected several relevant contextual and cultural factors. To begin, positive reactions to ADHD identification included agreement and motivation to seek help. It may be that our subsample of parents felt particularly motivated for care in the context of receiving the CLS school-based services, especially given service access and utilization barriers historically experienced by many Latino families in the U.S. (DeNavas-Walt, Proctor, \& Smith, 2009; Zambrana \& CarterPokras, 2004). Negative reactions from our subsample of Spanish-speaking Latino parents reflected difficulty accepting child psychopathology, which has been identified in previous research as particularly salient for Latino families (Gerdes et al., 2014), especially when ADHD behaviors are considered disrespectful (Perry et al. 2005). Interestingly, the importance of "respeto" or obedience to authority was described by many Spanish-speaking Latino parents in the current study, as well. Other reactions to ADHD identification from our-speaking Latino parents reflecting culture included the sentiment that Latinos are unfamiliar with ADHD, as well as the perception that hyperactivity is normal for children, especially boys. Although these perceptions could be interpreted as negative and reflective of disagreement in isolation, these statements in the current study were described matter-offactly and did not seem to carry a positive or negative sentiment in context of the larger 
interview/focus group. These perceptions related to culture in part may be explained by the influence of machismo or traditional gender roles often held by Latino families (Barker et al., 2010), as well as the lack of ADHD psychoeducation available in Latin countries (Palacios-Cruz et al., 2011). Overall, themes suggest that despite a lack of familiarity with ADHD in the Latino population, many families in our subsample of Spanish-speaking Latino parents described a desire to utilize ADHD services, especially if they address problems discordant with traditional values, such as respeto or respect for authority.

\section{Limitations and Future Directions}

These findings should be interpreted in light of some limitations, which also can serve as springboards for future research. The first limitation involves child and family differences between Latino and non-Latino families that may confound the quantitative results of this study. Latino families in our study reported lower primary parent education and household income, more single parent households, and higher receipt of public assistance than nonLatino families; Latino children showed significantly lower (albeit average) cognitive performance scores compared to non-Latino children (who also demonstrated average cognitive abilities). Such differences may make it more difficult to determine whether discrepancies between parent and teacher problem identification are due to cultural influences per se. However, strong support for the specificity of our results to the Latino population comes from secondary polynomial analyses controlling for child IQ, single parent status, and parent education. These analyses show a similar pattern of findings ${ }^{4}$, with attenuated significance values likely due to power restrictions potentially resulting from inclusion of additional covariates. There is a possibility that inclusion of covariates may have increased the precision of analyses; however, this is unlikely given that the majority of significance values in analyses with covariates remained in the trending or significant level and betas obtained were similar to betas from the main analyses.

Specific mechanisms contributing to the pattern of parent-teacher discrepancies found for Latino families in our study are unclear. We posit that parent-teacher communication may be an explanation for these findings based on previous studies indicating lower levels of parentschool involvement in Latino families (Wong \& Hughes, 2006). Unfortunately, direct measures of parent-teacher communication (e.g., quantity and quality of parent-teacher communications) were not available for this study. It would be important for future research to directly investigate whether parent-teacher disagreement for Latino families may be due to differences in their communications and/or language barriers. It will be useful for such studies to employ a longitudinal methodology to examine influences of these mechanisms. The current study used cross-sectional analyses, which limits interpretations of the

\footnotetext{
${ }^{4}$ Inclusion of the covariates of child IQ, single parent status, and parent education yielded results similar to findings without such covariates. Indeed, there was a non-significant trending caregiver- by teacher-rating interaction for ADHD symptom severity, $\beta=-.48$, $S E=.27, p=.07$, a non-significant trending caregiver- by teacher-rating interaction for hyperactivity/impulsivity symptoms, $\beta=-.60$, $S E=.31, p=.05$, a main effect of parent ratings of impairment on Latino status, $\beta=.55, S E=.25, p=.02$, a main effect of parent ratings of impairment on Latino status in older children, $\beta=1.05, S E=.41, p=.01$, and a non-significant trending parent- by teacherrating interaction for impairment in younger children, $\beta=-.94, S E=.48, p=.05$. No interactions were found for parent and teacher ratings of attention symptoms or analyses using direct classroom observations. Findings of ratings of symptom clusters in older children and inattention symptoms in younger children were consistent with the current study's analyses. However, the interaction of parent and teacher ratings of hyperactivity/impulsivity in younger children $(n=78)$ yielded statistically non-significant results, $\beta=-$. $78, S E=.52, p=.13$, likely due to power and sample size restrictions.
} 
differential pattern of findings between younger and older children. For instance, it cannot be determined that these age-related differences are due to maturation and improvements in parent-teacher communication across time. Furthermore, given that this study is one of the first to examine rater discrepancies with respect to a Latino population, our analyses were considered exploratory and thus were conducted without alpha level adjustments, which is an additional limitation. In addition, future research using equivalence tests for analyses of interactions between parent and teacher ratings with BOSS subscales may be helpful.

Regarding the qualitative analysis, the current study is limited by a modest sample size of eighteen Latino parents participating in Spanish-speaking cohorts of a school-based treatment study. Consequently, our subsample of parents may not be entirely representative of all Latino families in the U.S., especially those who are treatment naïve. For example, it is possible that parent responses were affected by the psychoeducation and support provided in the school-based treatment study; however, emerging themes from the current study generally are supportive of those emerging from mixed-method research with treatment naïve Latino families (e.g., Gerdes et al., 2014; Perry et al., 2005). Additionally, previous research has identified that saturation can occur with as few as twelve participants, and furthermore, the basic elements for meta-themes can occur with as few as six participants (Guest, Bunce, \& Johnson, 2006). Thus, our sample size of eighteen participants across three focus groups and seven individual interviews indeed allowed us to obtain saturation of themes. Nonetheless, exploring if themes emerge from a larger, more representative sample is an important future direction.

In addition, all parents participated in the focus group/interview after receiving the CLS intervention and providing follow-up data (i.e., ten from CLS and eight from BAU waitlist control condition). Specifically, the ten CLS parents responded to the focus group/interview immediately after receiving CLS and providing follow-up data; the eight BAU parents responded to the focus group/interview after being held on the waitlist, providing follow-up data, and then receiving the CLS intervention. We unfortunately do not have enough power to examine differences in experience for those receiving the intervention immediately versus after the waitlist control and we posit this would be an interesting future direction of study. Furthermore, our decision to only interview Latino parents in the Spanish-speaking CLS cohorts may have introduced bias related to acculturation, which in turn could increase those families' difficulties navigating mental health service systems for their children due to language barriers. Indeed, examination of acculturation ratings indicates that our subsample of Spanish-speaking Latino parents report higher affiliation with Latino compared to Anglo value and behavior orientation. Our sample of Latino parents participating in CLS also appears relatively highly educated compared to national averages for Latino educational attainment. Future research should examine if mixed-method findings from the current study emerge in studies with larger, more representative Latino samples. Lastly, we did not have sufficient sample size to consider within-group differences in identification of qualitative themes. The Latino population in the U.S. demonstrates rich heterogeneity based on factors, such as acculturation, country of origin, and socioeconomic status (González Burchard et al., 2005). Thus, future mixed-method research examining the potential influence of these factors to identification of ADHD behaviors, causes, and reactions to ADHD identification is warranted. 
Finally, it is important to acknowledge that all qualitative studies are influenced by the researchers' viewpoints and experiences. Efforts to mitigate this were taken by composing a linguistically and culturally diverse research team. Future studies from independent research groups with different and larger Latino participant samples would provide added confidence in the current study's emerging themes and interpretations.

\section{Integrated Summary and Recommendations}

The current mixed-method study results taken together with previous research suggest that Latino parents may be apt to recognize and express culturally relevant concerns over ADHD-related impairment, whereas concerns about ADHD symptoms overall may be less evident. Latino parents in our study appeared more likely to disagree with teachers regarding both impairment and the ADHD symptom cluster of hyperactivity/impulsivity for younger children compared to older children. Commonly identified social-emotional impairment from Latino parents in the current study may align with commonly identified beliefs that emotional/psychological characteristics cause ADHD. In addition, these themes may align with positive thoughts/emotions related to ADHD identification reflecting agreement and optimism, especially given that ADHD was identified in the context of a psychosocial treatment program. Concerns about academic impairment may align with beliefs about school causes and the theme reflecting the importance of respect for authority (such as teachers); concerns about home impairment may align with beliefs about family causes, as well as the theme reflecting guilt and denial in response to ADHD identification. Reports about symptoms may align with beliefs about biological/physical causes, as well as themes reflecting the level of ADHD familiarity in the Latino population and perceptions about hyperactivity as a normative manifestation of childhood. In summary, the current study has added to our field's growing body of problem recognition literature to suggest that Latino families may be influenced by a nuanced interweaving of perceptions regarding ADHD manifestations (including impairment and symptoms), causes (including psychological/ emotional, contextual, and biological factors), and reactions to ADHD identification.

The results of this study in context with previous work have important implications for clinical practice and research. To begin, ADHD assessment for diagnosis and treatment progress always should incorporate viewpoints from multiple informants, domains, and contexts. However, this appears particularly imperative when working with Latino families of young children, who may display significant discrepancies in parent-teacher reports of ADHD symptoms and impairment. In addition, to bridge parent and teacher perceptions of child ADHD-related difficulties, efforts to promote communication between teachers and Latino parents early in a child's education may be warranted. Indeed, it is possible that Latino families may be unfamiliar with ADHD terminology and services, but nevertheless may experience relief when ADHD is identified and subsequent motivation to access ADHD services. Psychological and school-based services for ADHD may be particularly wellaligned with cultural values and etiological beliefs often held by Latino families. Positivelyframed treatment goals based on domains the family finds concerning and is motivated to work on, such as respect and compliance to adult authority, may reinforce engagement and prevent dissatisfaction or dropout. 


\section{Acknowledgments}

Funding: This study was funded by a United States (U.S.) Department of Education Grant (R324A120358; PI: Pfiffner) and a Ruth L. Kirschstein National Research Service Award (NRSA) for Individual Postdoctoral Fellows (1F32MH101971-01; PI: Haack). This manuscript was prepared during Dr. Haack's NIH Fogarty Global Health Fellowship (NIH/FIC 5R25TW009343).

\section{References}

Alvarado, C; Modesto-Lowe, V. Improving Treatment in Minority Children With Attention Deficit/ Hyperactivity Disorder; Clinical Pediatrics. 2016. 9922816645517

Alvarado C, Modesto-Lowe V. 2017; Improving Treatment in Minority Children With Attention Deficit/Hyperactivity Disorder. Clinical Pediatrics. 56(2):171-176. DOI: 10.1177/0009922816645517 [PubMed: 27113035]

American Psychiatric Association. Diagnostic and Statistical Manual of Mental Disorders, 5th Edition: DSM-5. 5. Washington, D.C: American Psychiatric Publishing; 2013.

Arcia E, Fernández MC. 2003; From awareness to acknowledgment: The development of concern among Latina mothers of children with disruptive behaviors. Journal of Attention Disorders. 6(4): 163-175. DOI: 10.1177/108705470300600403 [PubMed: 12931074]

Barker CH, Cook KL, Borrego J Jr. 2010; Addressing Cultural Variables in Parent Training Programs With Latino Families. Cognitive and Behavioral Practice. 17(2):157-166. DOI: 10.1016/j.cbpra. 2010.01.002

Biederman J, Mick E, Faraone SV. 2000; Age-Dependent Decline of Symptoms of Attention Deficit Hyperactivity Disorder: Impact of Remission Definition and Symptom Type. American Journal of Psychiatry. 157(5):816-818. DOI: 10.1176/appi.ajp.157.5.816 [PubMed: 10784477]

Biederman J, Petty CR, Woodworth KY, Lomedico A, Hyder LL, Faraone SV. 2012; Adult outcome of attention-deficit/hyperactivity disorder: A controlled 16-Year follow-up study. The Journal of Clinical Psychiatry. 73(7):941-950. DOI: 10.4088/JCP.11m07529 [PubMed: 22901345]

Bussing DR, Zima BT, Gary FA, Garvan CW. 2003; Barriers to detection, help-seeking, and service use for children with ADHD symptoms. The Journal of Behavioral Health Services \& Research. 30(2):176-189. DOI: 10.1007/BF02289806 [PubMed: 12710371]

Creswell, JW; Clark, VLP. Designing and conducting mixed methods research. 2007. Retrieved from http://onlinelibrary.wiley.com/doi/10.1111/j.1753-6405.2007.00097.x/full

Cuellar I, Arnold B, Maldonado R. 1995; Acculturation rating scale for mexican americans-II: A revision of the original ARSMA scale. Hispanic Journal of Behavioral Sciences. 17(3):275-304. DOI: $10.1177 / 07399863950173001$

Curry LA, Nembhard IM, Bradley EH. 2009; Qualitative and Mixed Methods Provide Unique Contributions to Outcomes Research. Circulation. 119(10):1442-1452. DOI: 10.1161/ CIRCULATIONAHA.107.742775 [PubMed: 19289649]

Daley D, Birchwood J. 2010; ADHD and academic performance: why does ADHD impact on academic performance and what can be done to support ADHD children in the classroom? Child: Care, Health and Development. 36(4):455-464. DOI: 10.1111/j.1365-2214.2009.01046.x

DeNavas-Walt, C, Proctor, BD, Smith, JC. Income, poverty, and health insurance coverage in the United States: 2009. U.S. Census Bureau; 2009. Retrieved from http://www.census.gov/prod/ 2010pubs/p60-238.pdf

Eraldi RB, Mazzuca LB, Clarke AT, Power TJ. 2006; Service utilization among ethnic minority children with ADHD: A model of help-seeking behavior. Administration and Policy in Mental Health and Mental Health Services Research. 33:607-622. [PubMed: 16862391]

Fabiano GA, Pelham WE Jr, Waschbusch DA, Gnagy EM, Lahey BB, Chronis AM, et al. BurrowsMacLean L. 2006; A Practical Measure of Impairment: Psychometric Properties of the Impairment Rating Scale in Samples of Children With Attention Deficit Hyperactivity Disorder and Two School-Based Samples. Journal of Clinical Child \& Adolescent Psychology. 35(3):369-385. DOI: 10.1207/s15374424jccp3503_3 [PubMed: 16836475] 
Faraone SV, Asherson P, Banaschewski T, Biederman J, Buitelaar JK, Ramos-Quiroga JA, et al. Franke B. 2015; Attention-deficit/hyperactivity disorder. Nature Reviews Disease Primers. 15020

Faraone SV, Sergeant J, Gillberg C, Biederman J. 2003; The worldwide prevalence of ADHD: Is it an American condition? World Psychiatry. 2(2):104-113.

Gadow, KD, Sprafkin, J. Child Symptom Inventory. Stony Brook, NY: Checkmate Plus; 1994.

Gerdes AC, Lawton KE, Haack LM, Hurtado GD. 2013; Assessing ADHD in latino families: evidence for moving beyond symptomatology. Journal of Attention Disorders. 17:128-140. [PubMed: 22100735]

Gerdes AC, Lawton KE, Haack LM, Schneider BW. 2014; Latino Parental Help Seeking for Childhood ADHD. Administration and Policy in Mental Health and Mental Health Services Research. 41(4): 503-513. DOI: 10.1007/s10488-013-0487-3 [PubMed: 23539223]

González Burchard E, Borrell LN, Choudhry S, Naqvi M, Tsai HJ, Rodriguez-Santana JR, et al. Risch N. 2005; Latino Populations: A Unique Opportunity for the Study of Race, Genetics, and Social Environment in Epidemiological Research. American Journal of Public Health. 95(12):2161-2168. DOI: 10.2105/AJPH.2005.068668 [PubMed: 16257940]

Gradow, KD, Sprafkin, J. Childhood Symptom Inventory-4 screening and norms manual. Stony Brook, NY: Checkmate Plus; 2002.

Guest G, Bunce A, Johnson L. 2006; How many interviews are enough? An experiment with data saturation and variability. Field Methods. 18(1):59-82.

Haack, LM; Gerdes, AC; Lawton, KE; Schneider, BW. Understanding and Measuring Functional Impairment in Diverse Children With ADHD: Development of the ADHD-FX Scale With an AtRisk, Community Sample. Journal of Attention Disorders. 2014.

Halgunseth LC, Ispa JM, Rudy D. 2006; Parental control in Latino families: An integrated review of the literature. Child Development. 77(5):1282-1297. DOI: 10.1111/j.1467-8624.2006.00934.x [PubMed: 16999798]

Hinshaw S, Owens E, Zalecki C. 2012; Prospective follow-up of girls with attention-deficit/ hyperactivity disorder into early adulthood: continuing impairment includes elevated risk for suicide attempts and self-injury. Journal of Consulting and Clinical Psychology. 80(6):1041-1051. [PubMed: 22889337]

Hinshaw, SP, Scheffler, RM. The ADHD Explosion: Myths, Medication, Money, and Today's Push for Performance. Oxford University Press; 2014.

Knight GP, Gon ales NA, Saen DS, Bonds DD, German M, Deardorff J, et al. Updegraff KA. 2010; The Mexican American cultural values scales for adolescents and adults. The Journal of Early Adolescence. 30(3):444-481. DOI: 10.1177/0272431609338178 [PubMed: 20644653]

Laird RD, LaFleur LK. 2014; Disclosure and Monitoring as Predictors of Mother-Adolescent Agreement in Reports of Early Adolescent Rule-Breaking Behavior. Journal of Clinical Child \& Adolescent Psychology. 0(0):1-13. DOI: 10.1080/15374416.2014.963856

Laird RD, LaFleur LK. 2016; Disclosure and monitoring as predictors of mother-adolescent agreement in reports of early adolescent rule-breaking behavior. Journal of Clinical Child \& Adolescent Psychology. 45(2):188-200. [PubMed: 25470114]

Laird RD, Reyes ADL. 2012; Testing Informant Discrepancies as Predictors of Early Adolescent Psychopathology: Why Difference Scores Cannot Tell You What You Want to Know and How Polynomial Regression May. Journal of Abnormal Child Psychology. 41(1):1-14. DOI: 10.1007/ s10802-012-9659-y

Laird RD, Weems CF. 2011; The equivalence of regression models using difference scores and models using separate scores for each informant: Implications for the study of informant discrepancies. Psychological Assessment. 23(2):388. [PubMed: 21319905]

Lawton KE, Gerdes AC, Haack LM, Schneider B. 2014; Acculturation, cultural values, and Latino parental beliefs about the etiology of ADHD. Administration and Policy in Mental Health. 41(2): 189-204. DOI: 10.1007/s10488-012-0447-3 [PubMed: 23224619]

Leslie LK, Wolraich ML. 2007; ADHD service use patterns in youth. Journal of Pediatric Psychology. 32(6):695-710. DOI: 10.1093/jpepsy/jsm023 [PubMed: 17556401] 
Palacios-Cruz L, Peña Fdela, Valderrama A, Patiño R, Portugal C, Pamela S, Ulloa RE. 2011; Conocimientos, creencias y actitudes en padres mexicanos acerca del trastorno por déficit de atención con hiperactividad (TDAH). Salud Mental. 34(2):149-155.

Pastor PN, Reuben CA. 2005; Racial and ethnic differences in ADHD and LD in young school-age children: parental reports in the National Health Interview Survey. Public Health Reports. 120(4): 383-392. [PubMed: 16025718]

Perry CE, Hatton D, Kendall J. 2005; Latino parents' accounts of attention deficit hyperactivity disorder. Journal of Transcultural Nursing. 16(4):312-321. [PubMed: 16160193]

Power TJ, Eiraldi RB, Clarke AT, Mazzuca LB, Krain AL. 2005; Improving mental health service utilization for children and adolescents. School Psychology Quarterly. 20(2):187.

Reardon, T; Harvey, K; Baranowska, M; O'Brien, D; Smith, L; Creswell, C. What do parents perceive are the barriers and facilitators to accessing psychological treatment for mental health problems in children and adolescents? A systematic review of qualitative and quantitative studies; European Child \& Adolescent Psychiatry. 2017. 1-25.

Roberts RE, Alegria M, Roberts CR, Chen IG. 2005; Mental health problems of adolescents as reported by their caregivers. The Journal of Behavioral Health Services \& Research. 32(1):1-13. DOI: 10.1007/BF02287324 [PubMed: 15632794]

Rothe EM. 2005; Considering cultural diversity in the management of ADHD in Hispanic patients. Journal of the National Medical Association. 91:175-235.

Schmitz MF, Velez M. 2003; Latino cultural differences in maternal assessments of attention deficit/ hyperactivity symptoms in children. Hispanic Journal of Behavioral Sciences. 25(1):110-122. DOI: $10.1177 / 0739986303251700$

Shapiro, ES. Direct observation: manual for the Behavioral Observation of Students in Schools (BOSS) Academic Skills Problems. Workbook NY, NY: Guildford Press; 2004.

Sinkovics RR, Alfoldi EA. 2012; Progressive focusing and trustworthiness in qualitative research. Management International Review. 52(6):817-845.

Stevens J, Harman JS, Kelleher KJ. 2005; Race/ethnicity and insurance status as factors associated with ADHD treatment patterns. Journal of Child and Adolescent Psychopharmacology. 15(1):8896. DOI: 10.1089/cap.2005.15.88 [PubMed: 15741790]

Stewart M, Makwarimba E, Barnfather A, Letourneau N, Neufeld A. 2008; Researching reducing health disparities: Mixed-methods approaches. Social Science \& Medicine. 66(6):1406-1417. DOI: 10.1016/j.socscimed.2007.11.021 [PubMed: 18248867]

Sullivan-Bolyai S, Bova C, Harper D. 2005; Developing and refining interventions in persons with health disparities: The use of Qualitative Description. Nursing Outlook. 53(3):127-133. DOI: 10.1016/j.outlook.2005.03.005 [PubMed: 15988449]

Turgay A, Goodman DW, Asherson P, Babcock TF, Pucci ML, Barkley RA. 2012; Lifespan persistence of ADHD: the life transition model and its application. Journal of Clinical Psychiatry. 73(2):192201. [PubMed: 22313720]

Wechsler, D. Wechsler Abbreviated Scale of Intelligence. 2nd. San Antonio, TX: The Psychological Corporation; 2011.

Willcutt EG. 2012; The prevalence of DSM-IV attention-deficit/hyperactivity disorder: A metaanalytic review. Neurotherapeutics. 9:490-499. [PubMed: 22976615]

Willcutt EG, Nigg JT, Pennington BF, Solanto MV, Rohde LA, Tannock R, et al. Lahey BB. 2012; Validity of DSM-IV attention deficit/hyperactivity disorder symptom dimensions and subtypes. Journal of Abnormal Psychology. 121(4):991-1010. DOI: 10.1037/a0027347 [PubMed: 22612200]

Wolraich M, Lambert EW, Bickman L, Simmons T, Doffing MA, Worley KA. 2004; Assessing the impact of parent and teacher agreement on diagnosing attention-deficit hyperactivity disorder. Journal of Developmental \& Behavioral Pediatrics. 25(1):41-47. [PubMed: 14767355]

Wong SW, Hughes JN. 2006; Ethnicity and Language Contributions to Dimensions of Parent Involvement. School Psychology Review. 35(4):645-662. [PubMed: 18084632]

Woods DK. Transana Multiuser 3.02: A tool for the transcription and qualitative analysis of video and audio data. 
Zambrana RE, Carter-Pokras O. 2004; Improving health insurance coverage for Latino children: a review of barriers, challenges and State strategies. Journal of the National Medical Association. 96(4):508-523. [PubMed: 15101671] 


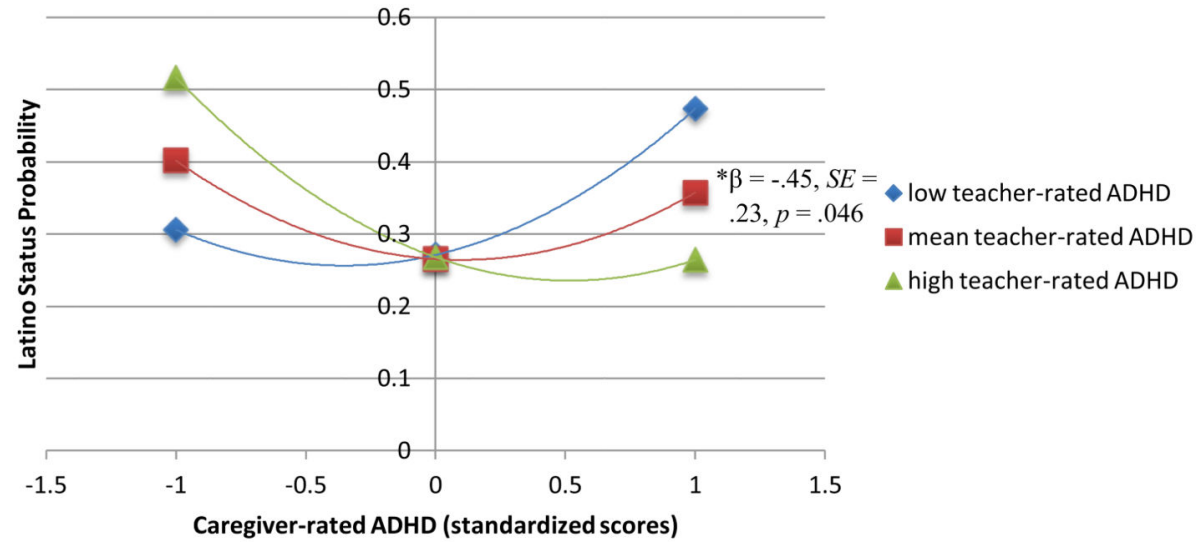

Figure 1. Probability of Latino status based on parent/caregiver \& teacher-rated ADHD across ages

*significant interaction 


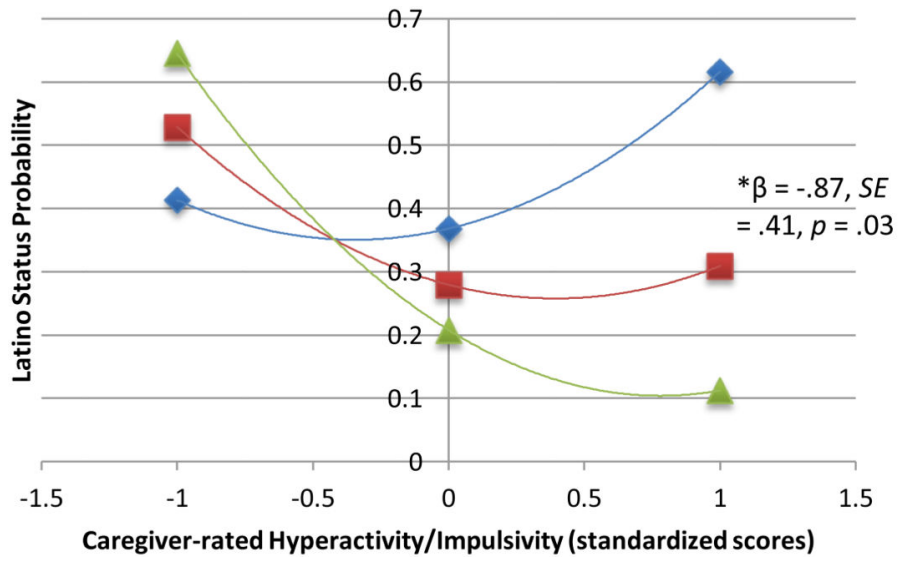

low teacher-rated hyperactivity/impulsivity

mean teacher-rated hyperactivity/impulsivity

$\Delta$ high teacher-rated hyperactivity/impulsivity

Figure 2. Probability of Latino status based on parent/caregiver \& teacher-rated hyperactivity/ impulsivity in younger children

*significant interaction 


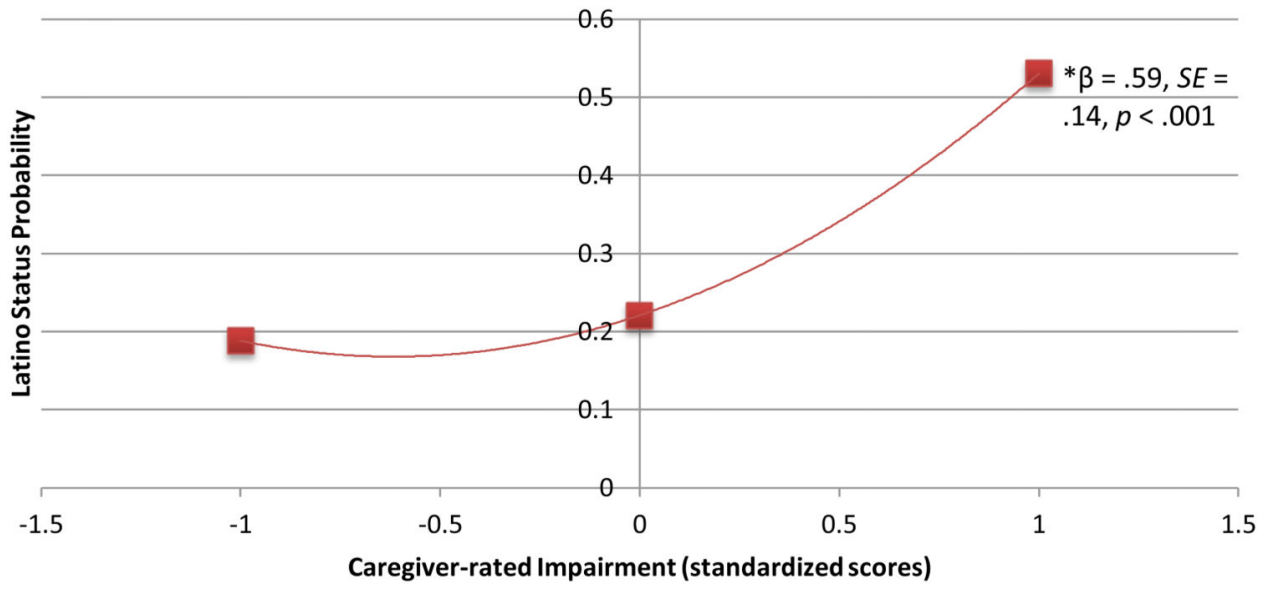

Figure 3. Probability of Latino status based on parent/caregiver \& teacher-rated impairment across ages

*quadratic main effect, no significant interaction 


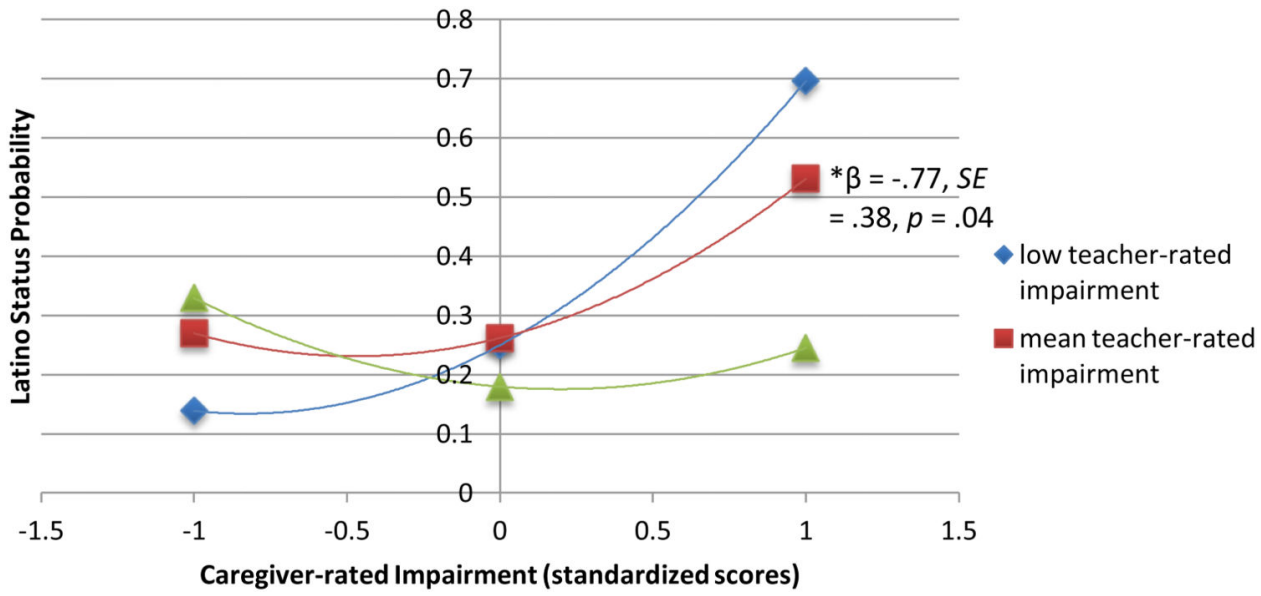

Figure 4. Probability of Latino status based on parent/caregiver \& teacher-rated impairment in younger children

*significant interaction 


\section{롤 \\ 골}
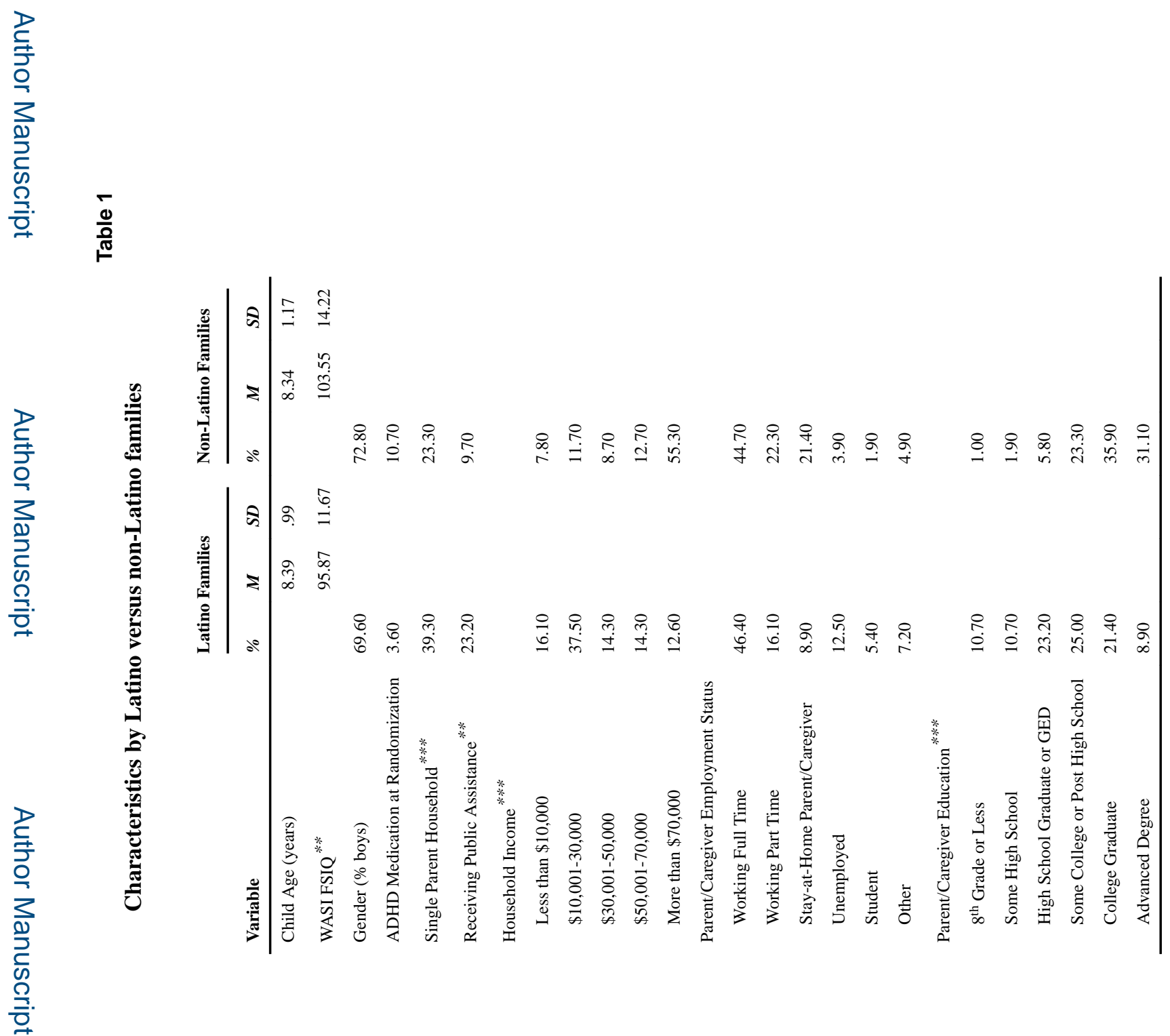

Adm Policy Ment Health. Author manuscript; available in PMC 2019 November 01. 


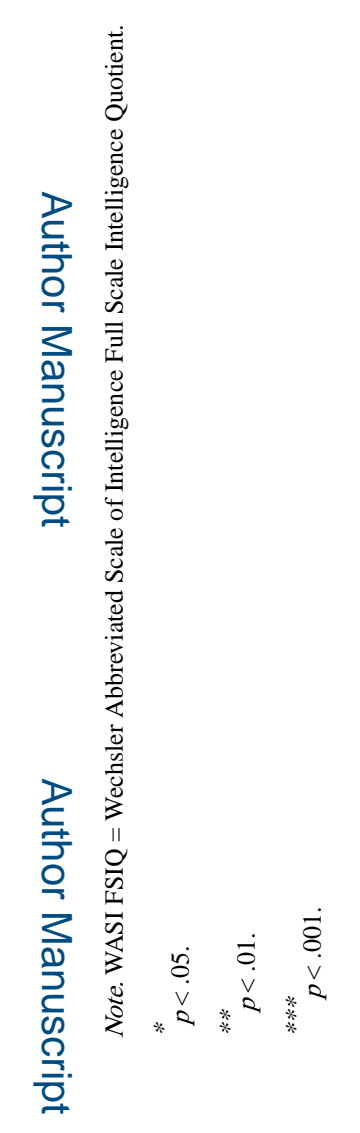

롤

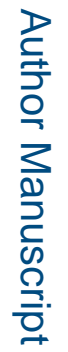

Adm Policy Ment Health. Author manuscript; available in PMC 2019 November 01. 
Table 2

Problem Recognition by Latino versus non-Latino families

\begin{tabular}{llllll} 
& \multicolumn{2}{c}{ Latino Families } & & \multicolumn{2}{c}{ Non-Latino Families } \\
\cline { 2 - 3 } Variable & $\boldsymbol{M}$ & $\boldsymbol{S D}$ & & $\boldsymbol{M}$ & \multicolumn{1}{c}{$\boldsymbol{S D}$} \\
\hline Parent/Caregiver-Rated ADHD & 31.47 & 11.77 & & 32.81 & 9.87 \\
Teacher-Rated ADHD & 33.00 & 10.38 & & 32.52 & 10.73 \\
Parent/Caregiver-Rated Inattention & 17.90 & 5.98 & & 18.32 & 4.72 \\
Teacher-Rated Inattention & 20.44 & 5.09 & & 19.06 & 5.31 \\
Parent/Caregiver-rated Hyperactivity/Impulsivity & 13.56 & 7.93 & & 14.62 & 7.24 \\
Teacher-rated Hyperactivity/Impulsivity & 12.40 & 7.90 & & 13.47 & 7.93 \\
Parent/Caregiver-Rated Impairment & 4.55 & 1.84 & & 4.20 & 1.25 \\
Teacher-Rated Impairment & 4.80 & 1.15 & & 4.62 & 1.32 \\
\hline
\end{tabular}

Note. $\mathrm{ADHD}=$ Attention-Deficit/Hyperactivity Disorder. 
Table 3

Acculturation characteristics of Latino families participating in Spanish-speaking CLS cohorts

\begin{tabular}{|c|c|c|c|}
\hline \multirow[b]{2}{*}{ Variable } & \multicolumn{3}{|c|}{ Latino Families } \\
\hline & $\%$ & $M$ & $S D$ \\
\hline \multicolumn{4}{|l|}{ MACVS Latino Orientation Subscales ${ }^{*}$} \\
\hline Familismo & & 4.30 & 0.58 \\
\hline Respeto & & 4.30 & 0.87 \\
\hline Traditional Gender Roles or Machismo & & 2.59 & 1.26 \\
\hline Religiosity or Spiritualismo & & 3.88 & 1.19 \\
\hline \multicolumn{4}{|l|}{ MACVS Anglo Orientation Subscales ${ }^{*}$} \\
\hline Material Success & & 1.80 & 0.87 \\
\hline Individualism and Self-Reliance & & 3.57 & 0.79 \\
\hline Competition and Personal Achievement & & 3.33 & 1.25 \\
\hline ARSMA-II Latino Orientation ${ }^{+}$ & & 3.92 & 0.86 \\
\hline ARSMA-II Anglo Orientation ${ }^{+}$ & & 3.13 & 1.18 \\
\hline \multicolumn{4}{|l|}{ ARSMA-II Acculturation Orthogonal Categories ${ }^{+}$} \\
\hline Bicultural (high Latino and Anglo orientation) & 12.00 & & \\
\hline Marginalized (low Latino and Anglo orientation) & 4.00 & & \\
\hline Assimilated (low Latino and high Anglo orientation) & 4.00 & & \\
\hline Separated (high Latino and low Anglo orientation) & 40.00 & & \\
\hline
\end{tabular}

Note. $\mathrm{N}=24$.

* Indicates missing data from one participant;

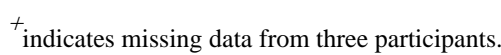

CLS = Collaborative Life Skills Program. MACVS = Mexican-American Cultural Values Scale for Adolescents and Adults (Knight et al,, 2010). ARSMA-II = Acculturation Rating Scale for Mexican Americans (Cueller et al., 1995). Both acculturation scales are rated on a 1 to 5 scale with higher values indicating higher affiliation/orientation. The ARSMA-II acculturation orthogonal categories are derived from the Latino and Anglo orientation scales, but not every participant score will be represented by a category; thus, percentages of ARSMA-II acculturation categories do not total $100 \%$. No group differences emerged on the ARMSA-II or MACV between Latino parents who did versus did not provide qualitative data. 
Table 4

Problem Recognition Qualitative Questions and Prompts

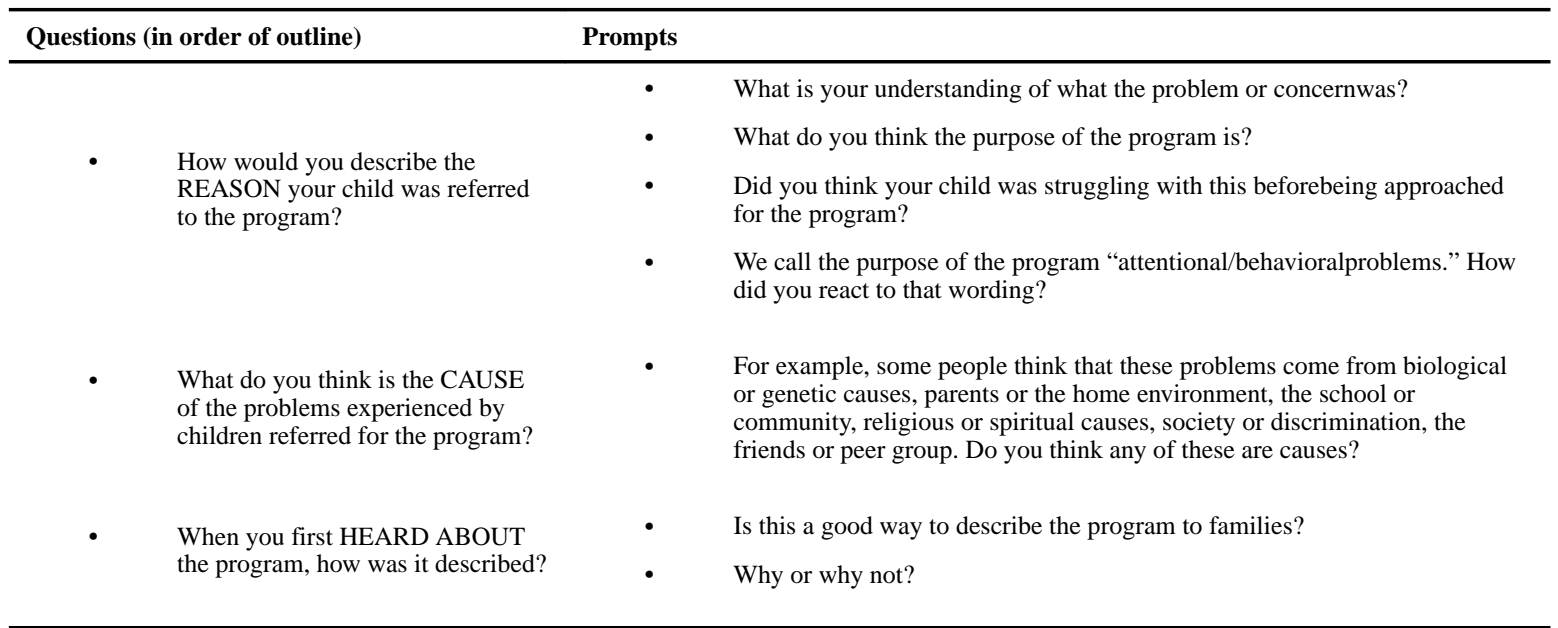


Table 5

Problem Recognition Identified in Qualitative Interviews/Focus Group

\begin{tabular}{|c|c|l|c|}
\hline \multicolumn{3}{|c|}{ Participants Identifying Social/Emotional Impairment; N (\%) } & \multicolumn{1}{|c|}{$\begin{array}{c}\text { Average Number of Codes Identified; M } \\
\text { (SD) }\end{array}$} \\
\hline \multicolumn{3}{|c|}{$13(\mathbf{7 2 \%})$} & \multicolumn{1}{c|}{ Example Quotes *translated from Spanish } \\
\hline Most Common Endorsed Codes & N (\%) & $7(39 \%)$ & -he was keeping it all inside, and there was a time when he exploded.* \\
\hline Doesn't express/show feelings appropriately & $4(22 \%)$ & $\begin{array}{l}\text {-with [my child], he likes to get the attention of other kids, \& he distracts } \\
\text { them.* }\end{array}$ \\
\hline Distractive, disruptive, bothersome to peers &
\end{tabular}

\begin{tabular}{|c|c|c|c|}
\hline \multicolumn{3}{|c|}{ Participants Identifying Academic Impairment (AC); N (\%) } & $\begin{array}{c}\text { Average Number of Codes Identified; } M \\
\text { (SD) }\end{array}$ \\
\hline \multicolumn{3}{|c|}{$11(61 \%)$} & $1.05(1.00)$ \\
\hline Most Common Endorsed Codes & $\mathbf{N}(\%)$ & \multicolumn{2}{|c|}{ Example Quotes *translated from Spanish } \\
\hline Behind academically/low grades & $6(33 \%)$ & \multicolumn{2}{|c|}{-because he couldn't keep up, he was delayed in some areas. * } \\
\hline Doesn't listen/pay attention in class ${ }^{\mathrm{a}}$ & $4(22 \%)$ & \multicolumn{2}{|c|}{-he doesn't pay attention in what the teacher is saying.* } \\
\hline \multicolumn{3}{|c|}{ Participants Identifying Home Impairment; N (\%) } & $\begin{array}{c}\text { Average Number of Codes Identified; } M \\
\text { (SD) }\end{array}$ \\
\hline \multicolumn{3}{|c|}{$8(44 \%)$} & $0.55(0.74)$ \\
\hline Most Common Endorsed Codes & $\mathbf{N}(\%)$ & \multicolumn{2}{|c|}{ Example Quotes *translated from Spanish } \\
\hline Doesn't finish/complete homework ${ }^{b}$ & $4(22 \%)$ & \multicolumn{2}{|c|}{$\begin{array}{l}\text {-he lied to me at the beginning. He said, "they didn't give me homework" or } \\
\text { "I lost my homework".* }\end{array}$} \\
\hline Creates stress for parents & $3(17 \%)$ & \multicolumn{2}{|c|}{$\begin{array}{l}\text {-sometimes it is not easy, to have a kid like that, because I am constantly } \\
\text { screaming at him; he has so much energy and doesn't stop. * }\end{array}$} \\
\hline
\end{tabular}

\begin{tabular}{|c|c|c|c|}
\hline \multicolumn{3}{|c|}{ Participants Identifying Inattention; N (\%) } & $\begin{array}{l}\text { Average Number of Codes Identified; } M \\
\text { (SD) }\end{array}$ \\
\hline \multicolumn{3}{|c|}{$13(72 \%)$} & $1.50(1.54)$ \\
\hline Most Common Endorsed Codes & $\mathbf{N}(\%)$ & \multicolumn{2}{|c|}{ Example Quotes *translated from Spanish } \\
\hline Easily distracted & $8(44 \%)$ & \multicolumn{2}{|c|}{$\begin{array}{l}\text { - his classmates are talking to him, when they are talking to him he is } \\
\text { distracted.* }\end{array}$} \\
\hline Trouble focusing & $6(33 \%)$ & \multicolumn{2}{|c|}{$\begin{array}{l}\text { - but she doesn't focus, she can watch the TV for } 10 \text { minutes and then start to } \\
\text { doing other thing or now she wants to do other thing and do this other thing.* }\end{array}$} \\
\hline
\end{tabular}

\begin{tabular}{|l|c|l|c|}
\hline \multicolumn{3}{|c|}{ Participants Identifying Hyperactivity/Impulsivity; N (\%) } & $\begin{array}{c}\text { Average Number of Codes Identified; M } \\
\text { (SD) }\end{array}$ \\
\hline \multicolumn{3}{|c|}{$\mathbf{5 ( 2 8 \% )}$} & \multicolumn{1}{c|}{$\mathbf{0 . 3 3 ( 0 . 5 9 )}$} \\
\hline Most Common Endorsed Codes & $\mathbf{N}(\%)$ & Example Quotes *translated from Spanish \\
\hline General hyperactive/restless -"inquieto" & $5(28 \%)$ & -he's so restless/inquieto. * \\
\hline Talks excessively & $2(11 \%)$ & -so, my daughter liked to talk a lot, she like to talk. * \\
\hline \multicolumn{3}{|c|}{ Participants Identifying ADHD or ADD; N (\%) } \\
\hline
\end{tabular}


Table 6

Etiology Identified in Qualitative Interviews/Focus Groups

\begin{tabular}{|c|c|c|c|}
\hline \multicolumn{3}{|c|}{ Participants Identifying BioPsychoSocial Causes; N (\%) } & Average Number of Codes Identified; M (SD) \\
\hline \multicolumn{3}{|c|}{$16(89 \%)$} & $0.89(0.32)$ \\
\hline $\begin{array}{l}\text { Most Common Endorsed } \\
\text { Codes }\end{array}$ & $\mathbf{N}(\%)$ & \multicolumn{2}{|c|}{ Example Quote *translated from Spanish } \\
\hline Psychological/emotional & $14(78 \%)$ & \multicolumn{2}{|c|}{$\begin{array}{l}\text {-so, I don't know the cause, I don't know if... I feel that it is something emotional or } \\
\text { psychological.* }\end{array}$} \\
\hline Familial/family Discord & $6(33 \%)$ & \multicolumn{2}{|c|}{-and even I think I had something to do with it. * } \\
\hline Educational & $5(28 \%)$ & \multicolumn{2}{|c|}{$\begin{array}{l}\text {-he didn't go to pre-k and that affected him. He didn't know how to read, lecture... When } \\
\text { he came to this school, he started on zero again. }\end{array}$} \\
\hline Physical/biological/Genetic & $5(28 \%)$ & \multicolumn{2}{|c|}{$\begin{array}{l}\text {-well I think they are born that way because of heredity. Because I remember that I was } \\
\text { like that, really mischievous too.* }\end{array}$} \\
\hline \multicolumn{3}{|c|}{ Participants Identifying Social/Spiritual Causes; N (\%) } & Average Number of Codes Identified; $M$ (SD) \\
\hline \multicolumn{3}{|c|}{$4(22 \%)$} & $0.22(0.43)$ \\
\hline Endorsed Codes & $\mathbf{N}(\%)$ & \multicolumn{2}{|c|}{ Example Quotes *translated from Spanish } \\
\hline Cultural Issue/acculturation & $3(17 \%)$ & \multicolumn{2}{|c|}{$\begin{array}{l}\text {-and here that the system is different, in my time I talked back to my mom and my "teeth } \\
\text { flew" [refers to her mom hitting her], here I can't do it [use physical discipline] and also it } \\
\text { is not good because that felt really bad but unfortunately I was raised in Mexico until I } \\
\text { was } 22 \text { years old, I had that education." }\end{array}$} \\
\hline
\end{tabular}


Table 7

Reactions to ADHD Identification Identified in Qualitative Interviews/Focus Groups

\begin{tabular}{|c|c|c|c|}
\hline \multicolumn{3}{|c|}{$\begin{array}{l}\text { Participants Identifying Positive Thoughts \& Emotions related to ADHD } \\
\text { Identification; N (\%) }\end{array}$} & Average Number of Codes Identified; $M(S D)$ \\
\hline \multicolumn{3}{|c|}{$3(17 \%)$} & $0.22(0.54)$ \\
\hline $\begin{array}{l}\text { Most Commonly Endorsed } \\
\text { Code }\end{array}$ & $\mathbf{N}(\%)$ & \multicolumn{2}{|c|}{ Example Quotes *translated from Spanish } \\
\hline Agree there's a problem & $2(11 \%)$ & \multicolumn{2}{|c|}{$\begin{array}{l}\text {-I knew I had to have a positive reaction, because they were helping me. They didn't } \\
\text { want to make something worse, or something bad for my child. Also, I needed to give } \\
\text { more attention to my child and help him. And they're helping me to do something } \\
\text { good.* }\end{array}$} \\
\hline \multicolumn{3}{|c|}{$\begin{array}{l}\text { Participants Identifying Negative Thoughts \& Emotions related to ADHD } \\
\text { Identification; N }(\%)\end{array}$} & Average Number of Codes Identified; $M(S D)$ \\
\hline \multicolumn{3}{|c|}{$6(44 \%)$} & $0.78(1.52)$ \\
\hline $\begin{array}{l}\text { Most Commonly Endorsed } \\
\text { Codes }\end{array}$ & $\mathbf{N}(\%)$ & \multicolumn{2}{|c|}{ Example Quotes *translated from Spanish } \\
\hline It was hard/difficult & $4(22 \%)$ & \multicolumn{2}{|l|}{-honestly, it was hard } \\
\hline Shock & $2(11 \%)$ & \multicolumn{2}{|c|}{$\begin{array}{l}\text { - but I was like, "really, my kid had that?" so it's kind of a shocker or a blow to the } \\
\text { parent like, "oh, do I have to go through that?" }\end{array}$} \\
\hline Guilt & $2(11 \%)$ & \multicolumn{2}{|c|}{ - I thought, "what did I do? What did I do differently? Is it my fault, really?"* } \\
\hline Denial & $2(11 \%)$ & \multicolumn{2}{|c|}{$\begin{array}{l}\text {-many people find difficult to accept culturally, it is culturally hard to accept that your } \\
\text { child has a problem* }\end{array}$} \\
\hline \multicolumn{3}{|c|}{$\begin{array}{l}\text { Participants Identifying Neutral Thoughts \& Emotions related to ADHD } \\
\text { Identification; N }(\%)\end{array}$} & Average Number of Codes Identified; $M(S D)$ \\
\hline \multicolumn{3}{|c|}{$6(44 \%)$} & $0.50(0.79)$ \\
\hline $\begin{array}{l}\text { Codes endorsed by at least } 1 \\
\text { caregiver }\end{array}$ & $\mathbf{N}(\%)$ & \multicolumn{2}{|c|}{ Example Quotes *translated from Spanish } \\
\hline "Respeto" is important & $4(22 \%)$ & \multicolumn{2}{|c|}{-the children need to follow the parent's rules, no matter what, without questions* } \\
\hline $\begin{array}{l}\text { High activity is considered } \\
\text { normal for kids, especially boys }\end{array}$ & $3(17 \%)$ & \multicolumn{2}{|c|}{$\begin{array}{l}\text {-I may notice he is active, he likes it, but they are children too, boys are different than } \\
\text { girls. I discovered it } 100 \% \text {, sometimes they are like more physical, they like to run, play } \\
\text { more, jump more, and girls no, they are more calm, but my girl looks like a boy.* }\end{array}$} \\
\hline $\begin{array}{l}\text { ADHD unfamiliar in Latino } \\
\text { culture }\end{array}$ & $2(11 \%)$ & \multicolumn{2}{|c|}{$\begin{array}{l}\text {-maybe because we have so little, you know, knowledge about ADHD or ADD, to hear } \\
\text { attention/behavioral problems, is there an understanding about it?** }\end{array}$} \\
\hline
\end{tabular}

